\title{
BMJ Open Comparing pharmacological venous thromboembolism prophylaxis to intermittent pneumatic compression in acute intracerebral haemorrhage: protocol for a systematic review and network meta-analysis
}

\author{
Vignan Yogendrakumar, ${ }^{1}$ Ronda Lun, ${ }^{1}$ Brian Hutton, ${ }^{2}$ Dean A Fergusson, ${ }^{2}$ \\ Dar Dowlatshahi ${ }^{1}$
}

To cite: Yogendrakumar V, Lun R, Hutton B, et al. Comparing pharmacological venous thromboembolism prophylaxis to intermittent pneumatic compression in acute intracerebral haemorrhage: protocol for a systematic review and network meta-analysis. BMJ Open 2018;8:e024405. doi:10.1136/ bmjopen-2018-024405

- Prepublication history and additional material for this paper are available online. To view these files, please visit the journal online (http://dx.doi. org/10.1136/bmjopen-2018024405).

Received 24 May 2018

Revised 7 September 2018

Accepted 28 September 2018

Check for updates

(C) Author(s) (or their employer(s)) 2018. Re-use permitted under CC BY-NC. No commercial re-use. See rights and permissions. Published by BMJ.

For numbered affiliations see end of article.

Correspondence to Dr Vignan Yogendrakumar; vyogendrakumar@toh.ca

\section{ABSTRACT}

Introduction Patients with an intracerebral haemorrhage are at increased risk of venous thromboembolism. Pharmacotherapy and pneumatic compression devices are capable of preventing venous thromboembolism, however both interventions have limitations. There are no head-to-head comparisons between these two interventions. To address this knowledge gap, we plan to perform a systematic review and network meta-analysis to examine the comparative effectiveness of pharmacological prophylaxis and mechanical compression devices in the context of intracerebral haemorrhage.

Methods and analysis MEDLINE, PUBMED, EMBASE, CENTRAL, ClinicalTrials.gov and the Internet Stroke Trials Registry will be searched with assistance from an experienced information specialist. Eligible studies will include those that have enrolled adults presenting with spontaneous intracerebral haemorrhage and compared one or more of the respective interventions against each other and/or a control. Primary outcomes to be assessed are occurrence of new venous thromboembolism (deep vein thrombosis and/or pulmonary embolism) and haematoma expansion, defined as a significant enlargement of baseline haemorrhage or new haemorrhage occurrence. Both randomised and non-randomised comparative studies will be included. Data on participant characteristics, study design, intervention details and outcomes will be extracted. Study quality will be assessed using the Cochrane Risk of Bias Tool and the Robins-I tool. Bayesian network metaanalyses will be performed to compare interventions based on all available direct and indirect evidence. If the transitivity assumption for network meta-analysis cannot be met, we will perform a qualitative assessment.

Ethics and dissemination Formal ethics is not required as primary data will not be collected. The findings of this study will be disseminated through conference presentations, and peer-reviewed publications. In an area of clinical practice where equipoise exists, the findings of this study may assist in determining which treatment intervention is most effective in venous thromboembolism prevention.
Strengths and limitations of this study

- In an area of study (haemorrhagic stroke) with limited head-to-head comparisons, we will use a Bayesian network analysis to compare two major interventions used in venous thromboembolism prevention.

- We will be able to comprehensively survey the literature supporting these interventions and identify areas where further study may be required.

- The nature of network meta-analysis allows for the comparison of multiple treatment combinations.

- The inclusion of non-randomised studies will reflect the real-world application of these interventions.

- The inclusion of non-randomised studies may increase the risk of bias and heterogeneity. We will use the Robins-I tool to determine which non-randomised studies are most suited for inclusion in our network meta-analysis. In addition, we will also perform an analysis involving randomised controlled trials only.

PROSPERO registration number CRD42018090960.

\section{INTRODUCTION}

Venous thromboembolism in intracerebral haemorrhage

Spontaneous intracerebral haemorrhage, the non-traumatic rupture of blood vessels within the brain, is the most devastating form of stroke seen worldwide. ${ }^{1}$ Mortality can be as high as $55 \%$ in the first month alone, and approximately $75 \%$ of survivors will suffer severe long-term disability and prolonged immobilisation. $^{2}$ To further complicate matters, patients with intracerebral haemorrhage are at an increased risk of venous thromboembolism (VTE). ${ }^{3}$ The rates of symptomatic deep 
vein thrombosis (DVT) in intracerebral haemorrhage varies from $3 \%$ to $7 \%$, and subclinical thrombotic events are reported to be as high as $17 \% .{ }^{45}$ The prophylactic prevention of this disorder is critical in preventing worsened outcomes. The two primary methods of prevention in use today are pharmacological agents and mechanical compression. ${ }^{6}$

\section{Pharmacological prophylaxis}

The most common agents include heparin, and low molecular weight heparin (LMWH) ${ }^{6}$ While commonly used to prevent VTE in ischaemic stroke, the use of pharmacological prophylaxis in the context of intracerebral haemorrhage has been an area of ongoing discussion and debate. The primary concern is that the use of these medications may result in worsened intracranial bleeding, termed haematoma expansion. As such, clinicians go to great lengths to mitigate this risk. ${ }^{7}$ Although trial data are limited, observational data have not shown an increased risk in haematoma expansion with the use of pharmacological antithrombotics. ${ }^{38}$ Regardless, the concerns of haematoma expansion have contributed to the infrequent use of these agents in clinical practice..$^{9}$

\section{Pneumatic compression devices}

Conventional compression stockings and hoses, graduated compression stockings and pneumatic compression devices (PCDs) are all used to varying degrees within clinical practice. Of the three, PCDs have emerged as the most effective in VTE prevention in a variety of clinical scenarios. ${ }^{10}$ Within the context of ischaemic stroke, PCDs have been found to be effective in preventing DVT/ pulmonary embolism (PE) when compared to standard compression stockings. ${ }^{11}$ The use of PCDs in the context of intracerebral haemorrhage is increasing in clinical practice ${ }^{12}$ However, trials displaying its effectiveness are limited in number. ${ }^{11} 13$

\section{Why is it important to do this review?}

Pharmacological methods and PCDs are capable of preventing VTE in patients with acute intracerebral haemorrhage. However, both interventions have limitations. To date, there have been no head-to-head comparisons of pharmacological prophylaxis and mechanical compression devices. This lack of comparative data have led to differences in guideline recommendations between varying organisations. The American Heart Association currently recommends the use of PCDs during initial presentation with potential to transition to systemic therapy after 1-4 days of symptom onset. ${ }^{6}$ In contrast, the European Stroke Organization does not provide any recommendations on when pharmacological agents should be used and instead recommends the use of PCD for patients with immobile haemorrhage. ${ }^{14}$

The aim of this study is to compare the effectiveness of pharmacological prophylaxis and intermittent PCDs in preventing VTE within the context of acute intracerebral haemorrhage. We will perform a systematic review and, because no major head-to-head comparisons of these two interventions currently exist, we will assess the effect of each intervention against control interventions and using a network meta-analysis (NMA), indirectly compare the two interventions. ${ }^{15-17}$

\section{METHODS}

\section{Study registration}

This study was registered with PROSPERO and will be conducted based on the guidelines of the Cochrane Handbook for Systematic Reviews. ${ }^{18}$ The findings of this study will be reported using the Preferred Reporting Items for Systematic Reviews and Meta-Analyses (PRISMA) extension statement for reporting of systematic reviews incorporating NMA. ${ }^{19}$ Any deviations incurred during performance of the systematic review will be described in the final report.

\section{Inclusion/exclusion criteria}

Eligibility criteria were established in terms of the Population-Intervention and Comparators-Outcomes-Study design framework. Studies will be selected according to the following criteria:

\section{Participants}

Included studies will involve adult patients $(\geq 18$ years of age) presenting with spontaneous intracerebral haemorrhage, confirmed with either CT or MRI. We will include patients who undergo emergent haematoma evacuation but patients who receive acute haemostatic agents such as recombinant factor VIIa will be excluded. We will include studies that assessed patients who had a stroke of any type so long as data pertaining to patients who had a haemorrhagic stroke are assessed separately. Patients with intracerebral haemorrhage who are part of a larger neurosurgical cohort may also be included if they are assessed separately. Studies that assessed traumatic intracerebral haemorrhage will not be included.

\section{Interventions/comparators}

We plan to assess all studies investigating short-term treatments used to prevent primary VTE following acute intracerebral hemorrhage. The most common agents in use today are heparin and LMWH agents, dalteparin, enoxaparin. However, we will not limit our analysis to these agents alone and will evaluate the prophylactic potential of less commonly used treatments (ie, fondaparinux). There will be no restrictions on dose, frequency, duration or route of administration. When possible, we will categorise the timing of anticoagulation start into three categories: early (within the first 48 hours of hospital presentation), mid (between days 2 and 4 of hospital presentation) or late (day 4 onwards). We will limit our inclusion to devices that provide pneumatic compression through sequential or intermittent means only. Conventional compression stockings and graduate compression stockings will not be considered an adequate intervention 
as previous studies have shown the superior effect of PCDs. ${ }^{112021}$ Studies that combine both therapies will also be included. A combined therapy will be formally defined as those in which patients receive pharmacological prophylaxis and mechanical compression simultaneously or in sequence. Studies that aim to assess secondary VTE prevention (prophylaxis after a VTE event has already occurred) will not be considered.

We will include studies that use no treatment/standard medical care, placebo or compression stockings/graduated stockings as a control therapy, as we anticipate these interventions may serve as an important link for the basis of indirect comparison analyses.

\section{Outcomes}

There are two primary outcomes for this study:

VTE-defined as the diagnosis of a DVT (symptomatic or asymptomatic) detected with compressible ultrasound (or equivalent technology based on the time of study) or the diagnosis of a pulmonary embolism by CT pulmonary angiography or ventilation-perfusion lung scanning. The exact time of VTE diagnosis is study dependent, but we would aim to look at the diagnosis of new VTE events, within the first 30 days of haemorrhage onset. We will specifically distinguish between studies in which screening tests were done and those in which only symptomatic patients were tested.

Haematoma expansion-defined as an enlargement of intracerebral haemorrhage volume or occurrence of new haemorrhage when comparing baseline volume at initial presentation to follow-up imaging at varying timepoints. We will report on significant expansion, as defined by the authors, occurring within the first 30 days of haemorrhage onset.

There is one secondary outcome measure:

Mortality-defined as death due to any cause occurring within the first year from haemorrhage onset.

Studies that report on either VTE (asymptomatic/ symptomatic DVT, PE), enlargement of baseline haematoma (or development of new haemorrhage) or mortality will be included for review.

\section{Study designs}

We will include studies that allow for comparison between two cohorts. This includes randomised controlled trials (RCTs), cluster RCTs, non-RCTs, prospective and retrospective comparative cohort studies. Single cohort studies, cross sectional studies, case series and case reports will be excluded. Studies presented in abstract form only will be excluded if we cannot acquire additional information from the study authors via correspondence. We will only include studies that are presented in English language due to constraints in translational resources.

\section{Information sources and search strategy}

Using keywords and MeSH terms relating to intracerebral haemorrhage, pharmacological prophylaxis and PCDs, the following four databases will be searched:
MEDLINE, EMBASE, PubMed, CENTRAL (1946 to March 2018). Additional searches of ClinicalTrials.gov, Clinical Trials Registry via the Internet Stroke Center and Google Scholar will also be conducted. We will scan the reference list of included studies or reviews identified through this search. Released abstracts from the last 5 years in the International Stroke Conference or European Stroke Organization Conference that have not been published in full manuscript form will be screened to ensure completeness. Only quantitative sources will be sought out. Major keywords were identified and used to build a search strategy. This search strategy was piloted by study author VY using MEDLINE. The same studies were successfully identified, and the search strategy was thereby finalised. The primary search strategy will be used for databases using an OVID interface (online supplementary appendix I). Modifications to the search strategy will be made for databases that do not use an OVID interface.

\section{Study records}

Data management

Database search results will be downloaded and imported to EndNote Reference Manager Software (Clarivate Analytics, Philadelphia, Pennsylvania, USA) and then transferred to Distiller Systematic Review Software (Evidence Partners, Ottawa, Ontario, Canada). After removal of duplicate results, citation titles and abstracts will be screened.

\section{Selection process}

Reviewers will independently screen articles in a two-level process. Level 1 will involve a title and abstract screening for potentially eligible studies. Studies that score a 'Yes' or 'Unsure' in this phase will be brought forward for fulltext (level 2) evaluation. Full-text screening will use a precreated article screening form (online supplementary appendix II). In the event of a disagreement between the two authors in either stage, a third party (DD) will adjudicate. The process of study selection will be described using a PRISMA flow diagram.

\section{Data collection process}

Reviewers will independently extract data from the included studies using an a priori designed data extraction form (online supplementary appendix III). The form will be piloted on a sample of three articles by the review team and refined prior to data collection of all remaining articles. We will collect data related to basic publication characteristics (eg, year and journal of publication, authorship list, funding), study populations (eligibility criteria as well as demographic and medical history measures including pre-existing medical conditions, clinical severity and haemorrhage characteristics), interventions compared (including pharmacological characteristics: dose, route, frequency and/or device characteristics), outcomes reported (including numbers of events and sample size per intervention group, as well as study-specific outcome definitions) and study design 
information (including presence/absence of randomisation, methods for patient enrolment, study follow-up information and other such details). After extraction is completed, authors will compare the collected data for each study, and any disputes will be settled by discussion or by consultation of a third party if necessary (DD).

\section{Risk of bias assessment (individual studies)}

Both randomised and non-randomised studies will be included in this systematic review. Risk of bias for all RCTs will be assessed using the Cochrane Collaboration Risk of Bias tool. ${ }^{22}$ We will contact the original study investigators for more information if required. The methodological quality of all non-randomised studies (non-randomised control trials, prospective/retrospective cohort studies) will be assessed with the Robins-I tool for cohort studies. ${ }^{23}$ Only studies that score high on qualitiy assessment will be included in our primary analysis. The evaluation of studies will be conducted independently by two reviewers. We will attempt to resolve disagreements by discussion and if required, a third author (DD) will be consulted to achieve consensus.

\section{Data synthesis}

We will perform Bayesian NMAs to compare the effectiveness of each intervention using WinBUGS (MRC Biostatistics Unit, Cambridge, UK) and NetMetaXL software. ${ }^{24}$ We will assess patient characteristics, study design information and event rates of the comparator arms to evaluate the appropriateness of the transitivity assumption, ensuring enough similarity between the included studies are sufficiently similar to allow for reliable data pooling ${ }^{25}$; this step will be especially important given that we anticipate identification of eligible RCTs as well as non-randomised studies. ${ }^{26}$ Individual patient data may be included based on availability. We will correct zero cells using an adjusted continuity correction factor of 0.5. Dichotomous data (occurrence of VTE, occurrence of haematoma expansion) will be reported in terms of ORs with corresponding 95\% CIs. All analyses will be performed using three chains of initial values, with totals of 50000 or more burn-in and sampling iterations. Adequacy of model fit will be assessed by comparing the posterior total residual deviance to the number of unconstrained data points (ie, the total number of intervention groups across studies) in each analysis, and comparisons between models (eg, fixed vs random effects models) will be based on the deviance information criterion (with lower values being preferred, and differences of five points or more indicating an important difference). Estimates of the between-study variance parameter will also be reported for random effects analyses. Model convergence of all NMAs will be assessed by inspection of Gelman-Rubin diagnostic plots. We will assess the consistency assumption of NMAs by also fitting corresponding unrelated means models and comparing their associated deviance information criterion (DIC) values with those from the primary NMAs based on consistency models; scatter plots of deviance residuals from both analyses to identify any outlying studies. NMAs will first be performed based on data derived purely from RCTs, and data from non-randomised studies will subsequently be added, and the analyses run a second time such that the effects of these data are apparent to readers.

If clinical and methodological heterogeneity between studies are found to be substantial, we will present pairwise meta-analysis only. If the transitivity assumption cannot adequately be met, a descriptive summary of study findings will be presented. If appropriate and feasible based on available data, we will further investigate sources of heterogeneity through subgroup analyses related to the following factors: start time of intervention (eg, early vs mid vs late), pharmacological agent and dose used, and baseline haemorrhage volume (small, $<10 \mathrm{~mL}$ vs moderate $10-30 \mathrm{~mL}$ vs large $>30 \mathrm{~mL}$ ). If possible, we will assess symptomatic VTE (clinically significant DVT or PE) separately.

\section{Meta-bias(es)}

For any RCTs included in our primary analysis, we will assess for potential selective reporting bias by looking for any published study protocols and ensuring that the outcomes presented in the protocol match that of the final report. In studies where a protocol is not available, we will compare the outcomes reported to what is stated in the methods section to ensure consistency. If sufficient studies are included, publication bias will be assessed by way of funnel plots.

\section{Confidence in cumulative evidence}

The quality of evidence for all outcomes will be judged using a framework developed by the Grading of Recommendations Assessment, Development, and Evaluation working group designed for rating the quality of effect estimates derived from a NMA. ${ }^{27}$ We will use a four-step process: (1) present direct and indirect treatment estimates (OR with $95 \%$ CIs); (2) rate the quality of direct and indirect treatment estimates; (3) present NMA estimates (pool of direct and indirect estimates, OR with 95\% CIs); and (4) rate the quality of NMA estimates.

\section{Patient and public involvement}

Because the collected data within this systematic review originates from previously published studies, patients and the general public were not involved in the development of the research question or choice of outcome measures that we wanted to assess.

\section{ETHICS AND DISSEMINATION}

The findings of this review and analysis may aid clinicians in day-to-day decision-making, assist in future guideline development and guide future research endeavours. We will therefore disseminate the findings of our work through conference presentations, the popular press and a peer-reviewed publication. 


\section{CONCLUSION}

We aim to compare the effectiveness of pharmacological prophylaxis and intermittent mechanical compressive devices in preventing VTE in acute intracerebral haemorrhage populations. The findings of this review and analysis may aid clinicians in day-to-day decision-making, assist in future guideline development and guide future research endeavours.

\section{Author affiliations}

${ }^{1}$ Ottawa Stroke Program, The Ottawa Hospital, University of Ottawa, Ottawa, Ontario, Canada

${ }^{2}$ Clinical Epidemiology Program, School of Epidemiology, Public Health and Preventative Medicine, Ottawa Hospital Research Institute, University of Ottawa, Ottawa, Ontario, Canada

Acknowledgements The authors would like to thank Lindsey Sikora of the University of Ottawa Health Sciences library for assisting with the refinement of the database search strategy.

Contributors VY is the guarantor. VY, RL, BH and DD drafted the manuscript protocol. VY, BH, DAF and DD contributed to the development of the selection criteria, article screening strategy, risk of bias assessment strategy and data extraction criteria. VY developed the search strategy. BH provided statistical expertise. All authors read, provided feedback and approved the final protocol.

Funding DD is supported by a Heart and Stroke Foundation New Investigator Award and a University of Ottawa Department of Medicine Clinician-Scientist Chair. $\mathrm{BH}$ is supported by a New Investigator award from the Canadian Institutes of Health Research and the Drug Safety and Effectiveness Network.

Competing interests None declared.

Patient consent Not required.

Ethics approval No local research ethics board approvals are required for this study as primary data collection is not taking place.

Provenance and peer review Not commissioned; externally peer reviewed.

Open access This is an open access article distributed in accordance with the Creative Commons Attribution Non Commercial (CC BY-NC 4.0) license, which permits others to distribute, remix, adapt, build upon this work non-commercially, and license their derivative works on different terms, provided the original work is properly cited, appropriate credit is given, any changes made indicated, and the use is non-commercial. See: http://creativecommons.org/licenses/by-nc/4.0/.

\section{REFERENCES}

1. Qureshi Al, Mendelow AD, Hanley DF. Intracerebral haemorrhage. Lancet 2009;373:1632-44.

2. Brouwers HB, Greenberg SM. Hematoma expansion following acute intracerebral hemorrhage. Cerebrovasc Dis 2013;35:195-201.

3. Faust AC, Finch CK, Hurdle AC, et al. Early versus delayed initiation of pharmacological venous thromboembolism prophylaxis after an intracranial hemorrhage. Neurologist 2017;22:166-70.

4. Balami JS, Buchan AM. Complications of intracerebral haemorrhage. Lancet Neurol 2012;11:101-18.

5. Kiphuth IC, Staykov D, Köhrmann M, et al. Early administration of low molecular weight heparin after spontaneous intracerebral hemorrhage. A safety analysis. Cerebrovasc Dis 2009;27:146-50.

6. Hemphill JC, Greenberg SM, Anderson CS, et al. Guidelines for the management of spontaneous intracerebral hemorrhage: a guideline for healthcare professionals from the American Heart Association/ American Stroke Association. Stroke 2015;46:2032-60.
7. Dowlatshahi D, Demchuk AM, Flaherty ML, et al. Defining hematoma expansion in intracerebral hemorrhage: relationship with patient outcomes. Neurology 2011;76:1238-44.

8. Wu TC, Kasam M, Harun N, et al. Pharmacological deep vein thrombosis prophylaxis does not lead to hematoma expansion in intracerebral hemorrhage with intraventricular extension. Stroke 2011;42:705-9.

9. Prabhakaran $\mathrm{S}$, Herbers $\mathrm{P}$, Khoury J, et al. Is prophylactic anticoagulation for deep venous thrombosis common practice after intracerebral hemorrhage? Stroke 2015;46:369-75.

10. Pavon JM, Adam SS, Razouki ZA, et al. Effectiveness of intermittent pneumatic compression devices for venous thromboembolism prophylaxis in high-risk surgical patients: a systematic review. $J$ Arthroplasty 2016;31:524-32.

11. Dennis M, Sandercock P, Reid J, et al. Effectiveness of intermittent pneumatic compression in reduction of risk of deep vein thrombosis in patients who have had a stroke (CLOTS 3): a multicentre randomised controlled trial. Lancet 2013;382:516-24.

12. Cherian LJ, Smith EE, Schwamm LH, et al. Current practice trends for use of early venous thromboembolism prophylaxis after intracerebral hemorrhage. Neurosurgery 2018;82:85-92.

13. Lacut K, Bressollette L, Le Gal G, et al. Prevention of venous thrombosis in patients with acute intracerebral hemorrhage. Neurology 2005;65:865-9.

14. Steiner T, Al-Shahi Salman R, Beer R, et al. European Stroke Organisation (ESO) guidelines for the management of spontaneous intracerebral hemorrhage. Int J Stroke 2014;9:840-55.

15. Lu G, Ades AE. Combination of direct and indirect evidence in mixed treatment comparisons. Stat Med 2004;23:3105-24.

16. Caldwell DM, Ades AE, Higgins JP. Simultaneous comparison of multiple treatments: combining direct and indirect evidence. $B M J$ 2005;331:897-900

17. Catalá-López F, Tobías A, Cameron C, et al. Network meta-analysis for comparing treatment effects of multiple interventions: an introduction. Rheumatol Int 2014;34:1489-96.

18. Collaboration TC. Cochrane handbook for systematic reviews of interventions version 5.1.0, 2011.

19. Hutton B, Salanti G, Caldwell DM, et al. The PRISMA extension statement for reporting of systematic reviews incorporating network meta-analyses of health care interventions: checklist and explanations. Ann Intern Med 2015;162:777.

20. Dennis M, Sandercock PA, Reid J, et al. Effectiveness of thigh-length graduated compression stockings to reduce the risk of deep vein thrombosis after stroke (CLOTS trial 1): a multicentre, randomised controlled trial. Lancet 2009;373:1958-65.

21. CLOTS (Clots in Legs Or sTockings after Stroke) Trial Collaboration. Thigh-length versus below-knee stockings for deep venous thrombosis prophylaxis after stroke: a randomized trial. Ann Intern Med 2010;153:553-62.

22. Higgins JP, Altman DG, Gøtzsche PC, et al. The Cochrane Collaboration's tool for assessing risk of bias in randomised trials. BMJ 2011;343:d5928.

23. Sterne JA, Hernán MA, Reeves BC, et al. ROBINS-I: a tool for assessing risk of bias in non-randomised studies of interventions. BMJ 2016;355:i4919.

24. Brown S, Hutton B, Clifford T, et al. A Microsoft-Excel-based tool for running and critically appraising network meta-analyses-an overview and application of NetMetaXL. Syst Rev 2014;3:110.

25. Cameron C, Fireman B, Hutton B, et al. Network meta-analysis incorporating randomized controlled trials and non-randomized comparative cohort studies for assessing the safety and effectiveness of medical treatments: challenges and opportunities. Syst Rev 2015;4:147.

26. Efthimiou O, Mavridis D, Debray TP, et al. Combining randomized and non-randomized evidence in network meta-analysis. Stat Med 2017;36:1210-26.

27. Puhan MA, Schünemann $\mathrm{HJ}$, Murad MH, et al. A GRADE Working Group approach for rating the quality of treatment effect estimates from network meta-analysis. BMJ 2014;349:g5630. 